\title{
Research into the inversion of the induced polarization relaxation time spectrum based on the uniform amplitude sampling method
}

\author{
Pu Zhang ${ }^{1} \cdot$ Sheng Wang ${ }^{1} \cdot$ Kai-Bo Zhou ${ }^{1} \cdot$ Li Kong $^{1} \cdot$ Hua-Xiu Zeng ${ }^{2}$
}

Received: 2 April 2015/Published online: 14 January 2016

(c) The Author(s) 2016. This article is published with open access at Springerlink.com

\begin{abstract}
The induced polarization relaxation time spectrum (RTS) reflects the distribution of rock pore size, which is a key factor in estimating the oil or water storage capacity of strata. However, as the data acquisition and transmission abilities of well logging instruments are much limited due to the underground environment, it is necessary to explore suitable sampling methods which can be used to obtain an accurate RST with less sampling data. This paper presents a uniform amplitude sampling method (UASM), and compares it with the conventional uniform time sampling method (UTSM) and logarithm time sampling method (LTSM) in terms of the adaptability to different strata, RTS inversion accuracy, and stratum vertical resolution. Numerical simulation results show that the UASM can obtain high inversion accuracy of RTS with different kinds of pore size distribution formation, with high dynamic ranges of pore size, and with a small number of sampling points. The UASM, being able to adapt to the attenuation speed of polarization curve automatically, thus has the highest vertical resolution. The inversion results of rock samples also show that the UASM is superior to the UTSM and LTSM.
\end{abstract}

Keywords Uniform amplitude sampling - Relaxation time spectrum - Stratum pore distribution - Induced polarization

Kai-Bo Zhou

zhoukb@hust.edu.cn

1 School of Automation, Huazhong University of Science and Technology, Wuhan 430074, Hubei, China

2 China Petroleum Logging Co., Ltd, Xi' an 710077, Shaanxi, China

Edited by Jie Hao

\section{Introduction}

The size distribution of rock pores is a key factor in determining the storage capacity of oil or water in the strata. Compared with the NMR logging mode, the IP logging mode has greater probing depth, higher signal-tonoise ratio, and less cost, so it has wider and more favorable application prospects (Titov et al. 2002, 2010; Wang 2004). The IP logging instrument can obtain the polarization decay potential (Guan et al. 2010; Li et al. 2011) which contains very rich underground information. The RTS inverted from the IP decay potential can be used to analyze the pore size distribution of the strata, and then to make assessments on the rock permeability, the amount of oil or water storage, and so on (Liu et al. 2014a, b; Rezaee et al. 2006; Binley et al. 2005; Zimmermann et al. 2008; Gurin et al. 2013; Beckett and Augarde 2013; Li et al. 2010). A lot of research work has been carried out on the RTS, including inversion algorithms (Moody and Xia 2004; Xiao et al. 2012; Chen et al. 2013; Jang et al. 2014; Florsch et al. 2014), determining the appropriate number of relaxation time constants and its distribution (Tong et al. 2004; 2005; Buecker and Hoerdt 2013; Revil et al. 2014). The results of this work have a certain relevance for the inversion of IP RTS (Liu et al. 2014a). However, the above research was mostly done in the laboratory, and very little was in the in situ environment (Nie et al. 1987; Wang et al. 2011).

Restricted by underground environments, data storage space, calculation ability, and data transmission ability of the logging instruments has been quite limited (Wang et al. 2010; Wu et al. 2009; Zhang et al. 2007), so it is practically meaningful to find a new downhole data acquisition mode by which relatively complete IP relaxation process can be represented with less acquired data. 
The conventional sampling method is UTSM which is based on equal time interval sampling. Although the amount of data can be reduced by increasing the sampling interval, it will lose high-frequency components which are used to accurately describe the fast change of polarizability. If the sampling interval is too short, the amount of data will be too large, and it will overload the data flow of the logging instrument. So UTSM is not practical in the applications of in situ well logging. LTSM takes sampling points based on logarithmic time interval, and can match the attenuation characteristics of polarization curve, so it greatly reduces the amount of sampling data. However, since the lithology of actual rock strata is complex, the length of IP relaxation process changes greatly. The LTSM can only be set to a fixed sampling length, which leads to the difficulty of improving the vertical resolution. This paper presents a new way of sampling-UASM, based on equal amplitude interval sampling, which is expected to describe the polarization decay curve accurately with less sampling data. We compare the three methods in terms of the adaptability of the downhole instrument to different pore sizes and distributions of strata, the relationship between number of sampling points and the inversion error, and the vertical resolution to different lithology of strata. Lastly, we give the most suitable number of sampling points by using UASM.

\section{The IP RTS and uniform amplitude sampling}

\subsection{IP discrete equations description and the RTS}

We assume that the porous medium has the same pore diameter, then the measured IP decay potential will satisfy a single exponential decay law (Tong and Tao 2007; Liu et al. 2015). The corresponding relationship between the relaxation time constant and the pore diameter is as follows:

$T=\frac{\Phi^{2}}{D}$

where $T$ is the relaxation time constant, $\Phi$ is the pore diameter, and $D$ is the diffusion coefficient of ions in the pore fluid. Equation (1) shows that the relaxation time constant and the pore diameter have one-to-one correspondence.

However, the actual strata contain a series of pores with different diameters. Therefore, the actual relaxation process represents a superposition of a series of relaxation processes with different time constants. The decay potential $V(t)$ is composed of a series of exponential functions with different relaxation time constants. In order to eliminate the influence of the exciting current on the measurement, the ratio of decay potential $V(t)$ measured after supply current is off and the potential $V_{\mathrm{p}}$ measured when polarization process approaches stability, is regarded as the polarizability decay curve, and it can be described as follows:

$\eta(t)=\frac{V(t)}{V_{\mathrm{p}}}=\int_{T_{\min }}^{T_{\max }} f(T) \exp \left(-\frac{t}{T}\right) \mathrm{d} T$

The corresponding discrete matrix equation is

$\eta_{m \times 1}=J_{m \times n} f_{n \times 1} \quad i=1,2, \ldots, m, \quad j=1,2, \ldots, n$

where, $J_{i j}=\exp \left(-\frac{t_{i}}{T_{j}}\right), J$ is the $m \times n$ dimensional coefficient matrix; $t_{i}$ is the time moment of measuring the IP decay signal; $T_{j}$ is the relaxation time constant; $\eta_{i}$ is the IP decay signal at the time moment $t_{i} ; f_{j}$ is the weight coefficient corresponding to $T_{j}$.

There is no exact solution for the discrete matrix equations, but we can use a damped least square method to obtain an approximate solution $f$ which makes the error $\|J f-\eta\|^{2}$ the smallest. In order to obtain the best leastsquares solution, the regularization method is used to seek the minimum value of $\|J f-\eta\|^{2}+\alpha^{2}\|f\|^{2}$. Assume

$$
\begin{aligned}
L= & \|J f-\eta\|^{2}+\alpha^{2}\|f\|^{2}=\sum_{i=1}^{m}\left[\sum_{j=1}^{n}\left(J_{i j} f_{j}\right)-\eta_{i}\right]^{2} \\
& +\alpha^{2} \sum_{j=1}^{n} f_{j}^{2}
\end{aligned}
$$

where $\alpha^{2}$ is a damping factor, which is used to control the weight of regularization term $\|f\|^{2}$ to the fitting term $\|J f-\eta\|^{2}$. Then calculate the extreme value point of Eq. (4), let

$$
\begin{aligned}
\frac{\partial L}{\partial f_{k}} & =2 \sum_{i=1}^{m}\left[J_{i k} \sum_{j=1}^{n}\left(J_{i j} f_{j}\right)\right]-2 \sum_{i=1}^{m}\left(J_{i k} \eta_{i}\right)+2 \alpha^{2} f_{k}=0, \\
k & =1,2, \ldots, n
\end{aligned}
$$

After reorganization, we can get

$$
\left(J^{\mathrm{T}} J+\alpha^{2} I\right) f=J^{\mathrm{T}} \eta
$$

Then the least squares solution can be obtained as

$f=\left(J^{\mathrm{T}} J+\alpha^{2} I\right)^{-1} J^{\mathrm{T}} \eta$

Because $f_{j}$ cannot be negative, we put non-negative constraints on Eq. (6). Calculate repeatedly until the solution meets the condition $f_{j} \geq 0, j=1,2, \ldots, n$. The specific calculation steps are as follows:

Step 1 Calculate the initial solution $f^{k}(k=0)$ according to Eq. (7);

Step 2 Calculate the polarizability $\eta^{\prime}=J f^{k}$ according to Eq. (3); 
Step 3 Calculate the polarizability bias $\Delta \eta=\eta-\eta^{\prime}$;

Step 4 Obtain the solution's correction $\Delta f$ with $\Delta \eta$ according to Eq. (7);

Step 5 Correct the spectral components according to the equation $f^{k+1}=f^{k}+\Delta f$;

Step 6 If every component of the solution $f^{k+1}$ meets the constraint $f_{j} \geq 0$, then $f^{k+1}$ is the expected solution. Otherwise, set the negative component to zero, then let $\eta=\eta^{\prime}$, and then repeat step 2 to step 6 until the solution $f^{k+1}$ meets the requirement or the $k$ reaches the set iteration number.

\subsection{Uniform amplitude sampling}

The IP potential curve, after spontaneous potential compensation, is shown in Fig. 1, where $I_{0}$ is the supplied current, $V_{\mathrm{p}}^{+}$is the potential when the IP potential excited by positive current achieves a stable state, $V_{\mathrm{t}}^{+}(t)$ is the IP decay potential after the positive current is switched off. $V_{\mathrm{p}}^{-}$is the potential when the IP process excited by negative current achieves stability, $V_{\mathrm{t}}^{-}(t)$ is the IP decay potential after the negative current is switched off.

In the beginning after the current is switched off, the IP potential curve usually has a high decay speed and as time goes on the decay speed slows down. If we select the UTSM, it will be very difficult to solve the contradiction between the accuracy of the IP decay curve and the small number of sampling points. Since the UASM can track the change of decay voltage as shown in Fig. 2c, it can provide better accuracy with small sample numbers. Although LTSM matches the decay curve, it takes a longer sampling time compared with UASM as shown in Fig. 2b, c.

The polarization decay curve is drawn again in Fig. 3 with the timeline in logarithmic coordinates. If the number of sampling points is $m$, then the normalized equal-interval amplitude scale is obtained as $\left\{A_{i}\right\}=\left(1, \frac{m-1}{m}, \ldots\right.$, $\left.\frac{m-i+1}{m}, \ldots, \frac{2}{m}, \frac{1}{m}\right), i=1,2, \ldots, m$. And then the time signal

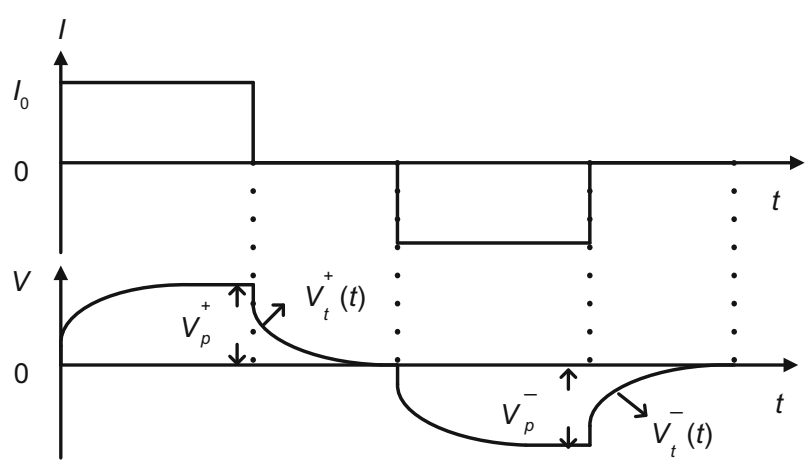

Fig. 1 Induced polarization potential curve
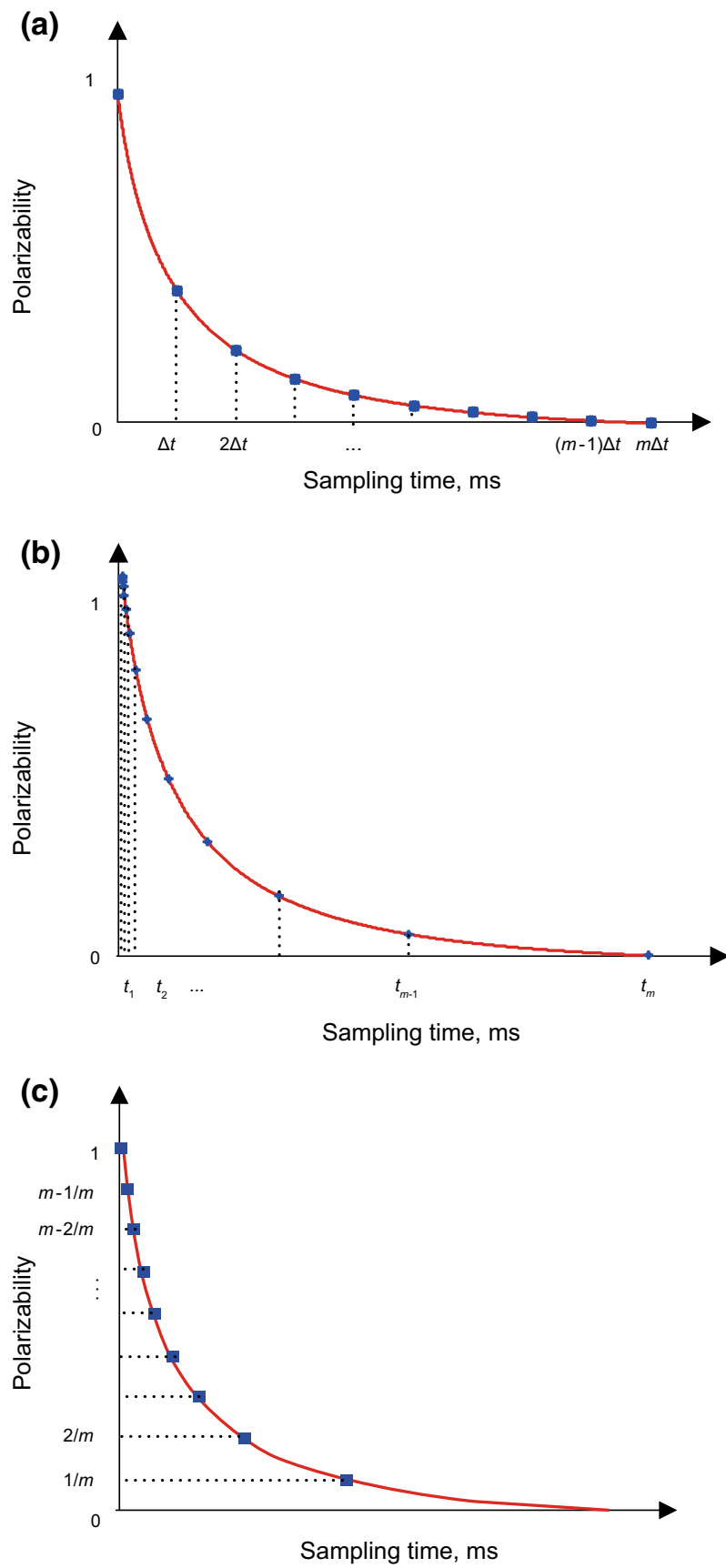

Fig. 2 Sampling methods for the polarization decay curve. a Uniform time sampling, b Uniform logarithm sampling, c Uniform amplitude sampling

sequence according to the corresponding amplitude scale can be determined as shown in Fig. 3.

Since in the actual measuring process, there is disturbance at the moment when supply current is switched off, the logging instrument usually begins to acquire polarization decay signals after a short period of pause. The A/D convertor of the instrument works at a fixed high frequency, but not all the sampled signal data will be saved. 


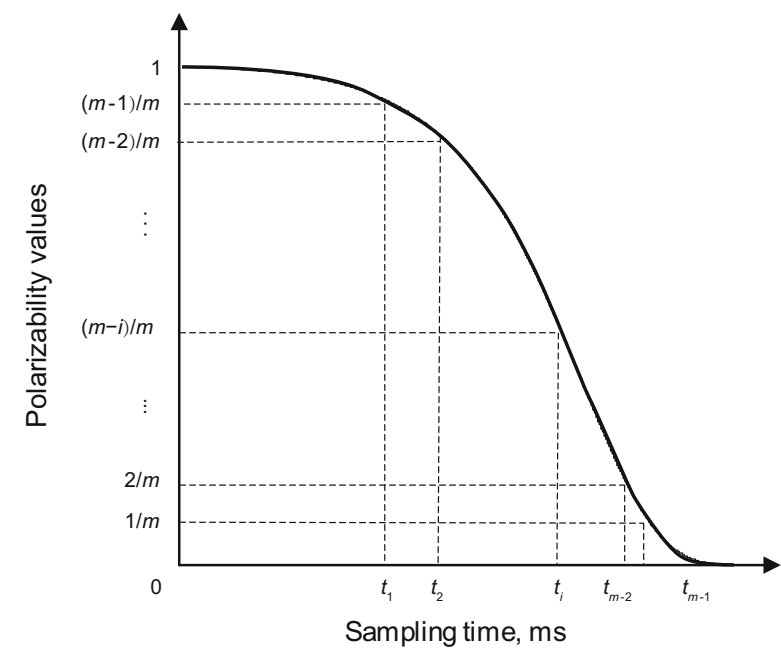

Fig. 3 Uniform amplitude sampling of the normalized polarizability curve

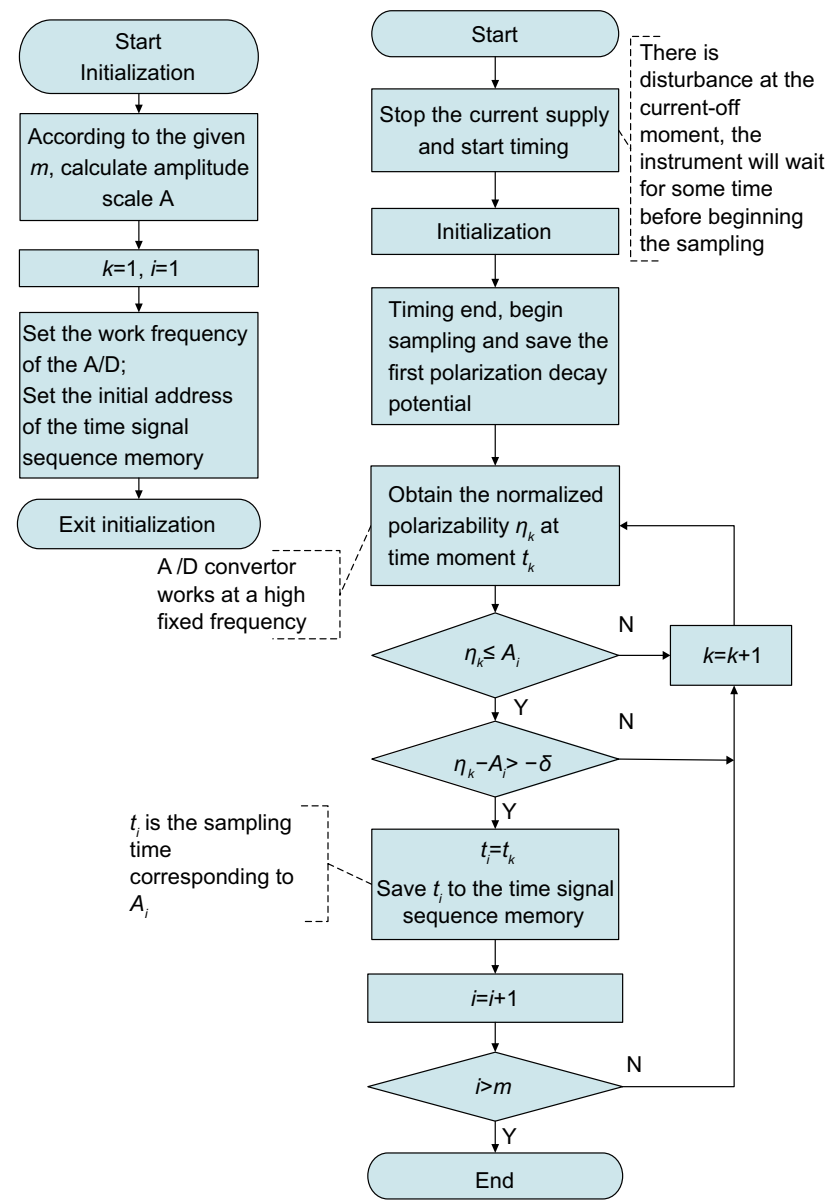

Fig. 4 Operation flow diagram of uniform amplitude sampling

Suppose the first time moment of A/D conversion of the IP decay potential be the zero of the time signal. The first $\mathrm{A} / \mathrm{D}$ converted value is saved in the register, and is set as a standard for normalizing each follow-up A/D conversion value. We compare the sampled value of normalized polarization decay potential with amplitude scale value $A_{i}$. If the sampled value is bigger than $A_{i}$, the sampling time will not be saved, and the measurement process goes into the next $\mathrm{A} / \mathrm{D}$ conversion cycle. If the sampled value is not bigger than $A_{i}$, and meets the condition $\eta_{k}-A_{i}>-\delta$, the sampling time is saved as the $i$ th time signal $t_{i}$ in the corresponding time signal sequence memory. If the sampled value does not meet the above condition, that value is set as interference, then this sampling time is not saved, and the measurement process goes into the next A/D conversion cycle. The process goes on until all the time signal values corresponding to the $m$ amplitude scale values are recorded in turn. The specific uniform amplitude sampling operation flow diagram is shown in Fig. 4.

As the UASM is very sensitive to the kind of disturbance which causes the amplitude to decrease sharply, while the actual normalized polarizability signal between two consecutive A/D values is far less than $\frac{1}{m}$, we set a threshold $\delta<\frac{1}{m}$. Only when the normalized polarizability value $\eta_{k}$ meets the condition of $-\delta<\eta_{k}-A_{i} \leq 0$, the sampling time is regarded as time signal $t_{i}$ corresponding to $A_{i}$, and it is then saved. Setting of this condition can completely inhibit the disturbance which led to a decrease of the amplitude greater than $\delta$ in the sampling process.

\section{The simulation}

\subsection{The comparison of the three sampling methods}

Since the lithology of actual rock strata is complex, and the rock pore sizes of different regions are very different (Yang et al. 2014; Zhu et al. 2014) even in the same well, the rock pore sizes of different depths may also be very different (Tan et al. 2010; Shou and Zhu 1998). We hope that the IP logging instrument can adapt to a wide range of strata with different pore diameters, and obtain higher inversion accuracy of the polarization relaxation time spectrum from a smaller number of sampling points. In order to compare the relative merits of the three sampling methods, we designed several relaxation spectrum models which meet the logarithm normal distribution, and do research on sampling of the polarization curve and on inverting the polarization relaxation time spectra of these models.

\subsubsection{Single-peak model}

Two kinds of single-peak relaxation time spectra are set as shown in Fig. 5. The relaxation time constants of model A are small, corresponding to the small diameters of the rock 

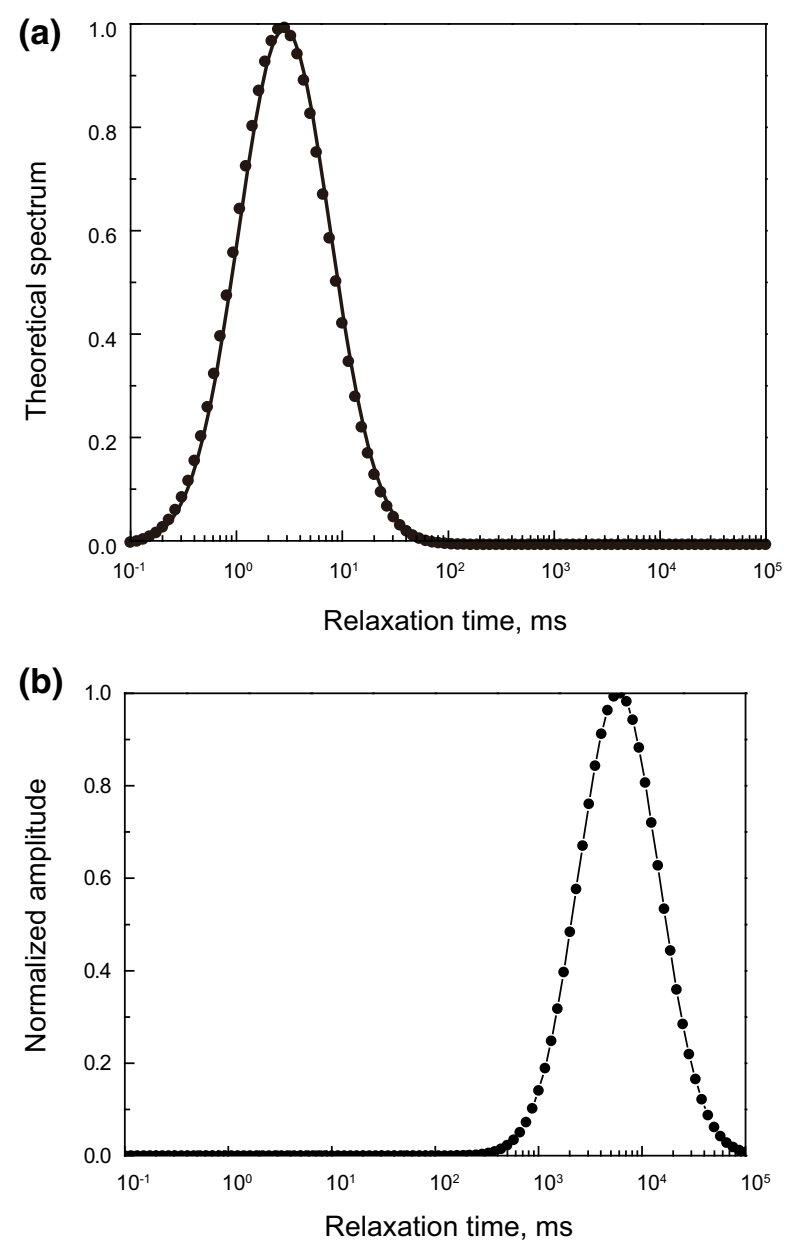

Fig. 5 The models of single-peak relaxation spectra. a Model A, b Model B

pores. The relaxation time constants of model B are large, corresponding to the large diameters of the rock pores. Polarization decay curves of two models are shown in Fig. 6, indicating that the curve of model A decays fast and the curve of model B decays slowly.

Assume that the sampling lengths of the two corresponding decay curves are both $100,000 \mathrm{~ms}$, and the numbers of sampling points are set as 30, 60, 100, 200, 300 , respectively; the three sampling methods will be used to sample the two corresponding decay curves. Suppose the relaxation time constants following a uniform logarithmic distribution between $0.1 \mathrm{~ms}$ and $100,000 \mathrm{~ms}$ be 100 for the above five situations, the relaxation time spectra can be inverted as shown in Figs. 7 and 8 with the nonnegative damping least squares method.

The root mean square error (RMSE) of inversion varies with the number of sampling points as shown in Tables 1 and 2 .

The logging instrument usually rises from the well bottom to the surface at a constant speed when measuring

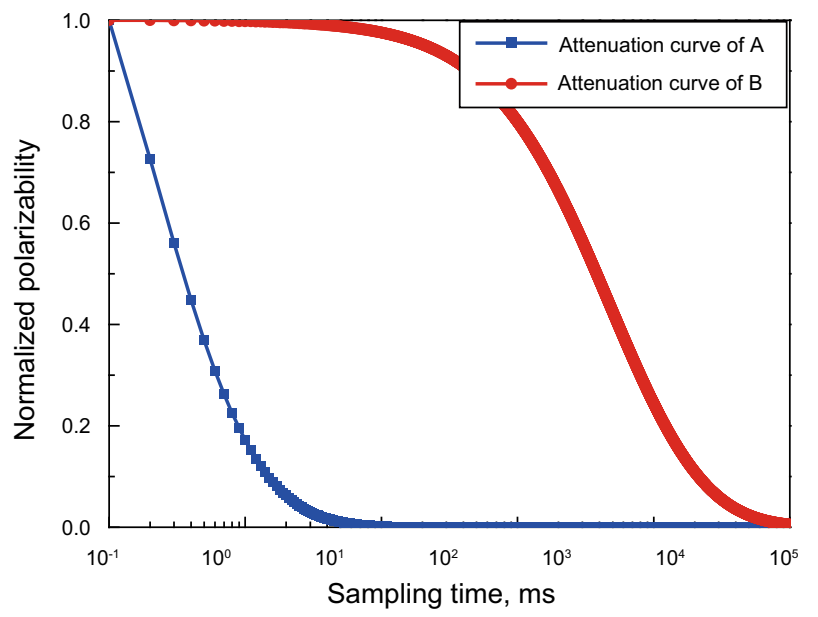

Fig. 6 Polarization decay curves of model A and model B

strata, thus the shorter the sampling time is, the thinner the corresponding stratum is, and the thinner the measured stratum is, the higher the vertical resolution will be. When using UTSM and LTSM, the sampling time is set as $100,000 \mathrm{~ms}$. However when using UASM, the data acquisition process ends once the last time point is recorded. The actual sampling time of UASM is shown in Table 3.

Figures 7, 8 and Tables 1, 2 show that, with the UTSM, the inversion accuracy of RTS is high only when the pore diameter of the rock formation is big. With the LTSM and UASM, the inversion accuracy of RTS is high with both big and small pore diameters of rock formation, and meanwhile the required accuracy can be approached with less sampling points. Table 3 shows that the UASM can track the amplitude variety of the polarizability curve very well, and thus it has the best vertical resolution among the three methods.

\subsubsection{Double-peak model}

In order to verify whether the above result is universal, we simulated the double-peak spectrum models C, D, and E over a wide range of pore diameter distribution. The models are shown in Fig. 9, where model $\mathrm{C}$ has more small pores and model D has more large pores, the high peak amplitude is about twice as high as the low peak amplitude. Model E has the same height of peak amplitude, but its pore size distribution is not continuous and the mean size values of the peaks are very different.

The simulation conditions are the same as single-peak models, and the simulation results are shown in Figs. 10, 11, and 12. The inversion errors are shown in Tables 4, 5, 

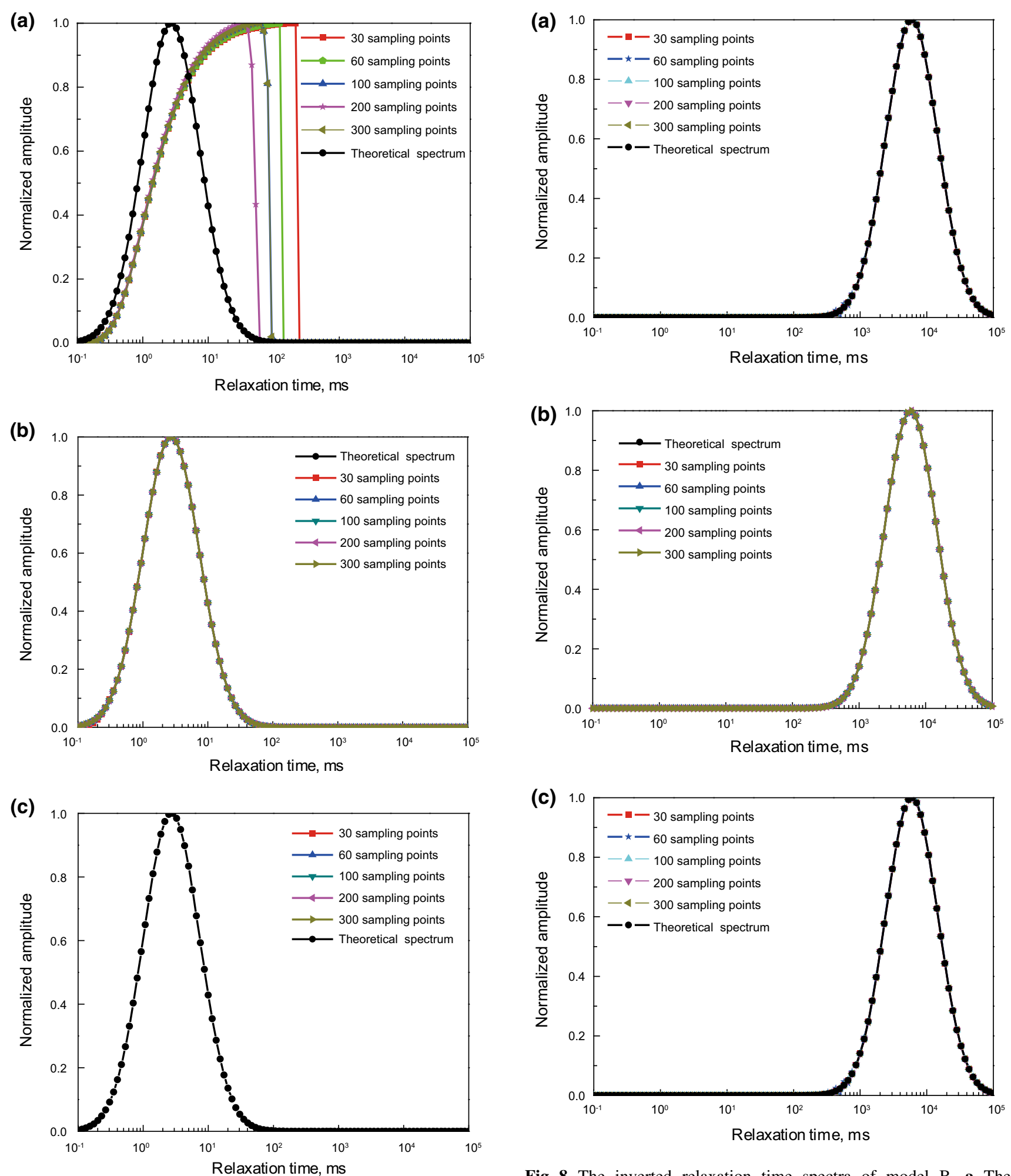

Fig. 7 The inverted relaxation time spectra of model A. a The relaxation time spectrum with the UTSM, b The relaxation time spectrum with the LTSM, c The relaxation time spectrum with the

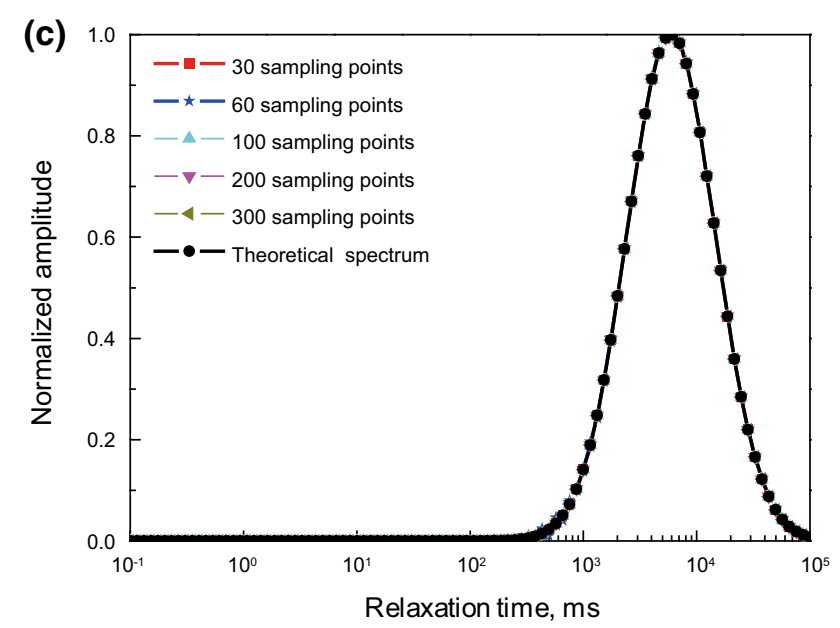

Fig. 8 The inverted relaxation time spectra of model B. a The relaxation time spectrum with the UTSM, b The relaxation time spectrum with the LTSM, c The relaxation time spectrum with the UASM 
Table 1 The RMSE of model A's inversion with three sampling methods

\begin{tabular}{llllll}
\hline Sampling points & 30 & 60 & 100 & 200 & 300 \\
\hline UTSM & 0.292 & 0.28 & 0.266 & 0.245 & 0.23 \\
LTSM & $2.17 \times 10^{-3}$ & $1.76 \times 10^{-4}$ & $3.19 \times 10^{-4}$ & $2.19 \times 10^{-4}$ & $3.18 \times 10^{-5}$ \\
UASM & $3.1 \times 10^{-4}$ & $1.1 \times 10^{-4}$ & $4.5 \times 10^{-5}$ & $2.5 \times 10^{-4}$ & $7 \times 10^{-5}$ \\
\hline
\end{tabular}

Table 2 The RMSE of model B's inversion with three sampling methods

\begin{tabular}{llllll}
\hline Sampling points & 30 & 60 & 100 & 200 & 300 \\
\hline UTSM & $2.1 \times 10^{-3}$ & $7.8 \times 10^{-4}$ & $2.6 \times 10^{-4}$ & $1.3 \times 10^{-4}$ & $1.5 \times 10^{-4}$ \\
LTSM & $3.33 \times 10^{-5}$ & $5.24 \times 10^{-6}$ & $1.6 \times 10^{-4}$ & $2.18 \times 10^{-4}$ & $1.13 \times 10^{-4}$ \\
UASM & $1.4 \times 10^{-4}$ & $1.6 \times 10^{-4}$ & $1.25 \times 10^{-4}$ & $1.8 \times 10^{-4}$ & $1.6 \times 10^{-4}$ \\
\hline
\end{tabular}

Table 3 Sampling time of model A and model B with UASM

\begin{tabular}{|c|c|c|c|c|c|}
\hline Sampling points & 30 & 60 & 100 & 200 & 300 \\
\hline Model A, ms & 38 & 52 & 63 & 70 & 77 \\
\hline Model B, ms & 54,298 & 74,339 & 87,897 & 98,373 & 84,532 \\
\hline
\end{tabular}

and 6. The actual sampling time of model C, D, and E with UASM is shown in Table 7.

Figures 10, 11, 12, and Tables 4, 5, and 6 show that there exists a large deviation between the inverted spectrum and the original theoretical spectrum using the UTSM. The deviation is especially larger for models $\mathrm{C}$ and E which have more small pores. However, by using the LTSM and the UASM, the inversion results are in good agreement with the theoretical spectrum for all three models. Table 7 shows that the actual sampling time decreases a lot when using UASM, which means that the logging instrument can measure more samples and improve the vertical resolution.

The simulation results of the double-peak spectrum are consistent with those of the single-peak spectrum, which can prove that the results in Sect. 3.1.1 have a certain universality. All the above simulation results of the rock formation models show that the UASM is better than the UTSM and the LTSM.

\subsection{Determine the best number of sampling points when using UASM}

We hope to get a qualified RTS with few sampling points. It can be found in the simulation in Sect. 3.1 that with the UASM, when the sampling point number is more than 100 , the inversion accuracy will not increase significantly. Therefore, the sampling point numbers are set as 15, 30, $40,50,60$, and 80 , respectively, in the following simulations. Since the actual pore size of strata mostly follows a multi-peak distribution, then a double-peak model $\mathrm{F}$, a three-peak model $\mathrm{G}$, and a four-peak model $\mathrm{H}$ are set as the simulation objects. The inverted spectra are shown in Fig. 13.

It is difficult to determine which number of sampling points is the best by observing the inverted spectra, so we calculate the RMSEs of the inverted spectra with different numbers of sampling points, and the results are shown in Fig. 14.

Figure 14 shows that the inversion error decreases slowly when the number of sampling points is more than 30 , and the inversion error may also fluctuate with the increase of the number of sampling points. Meanwhile, overmuch sampling will increase the data transfer burden and inversion computation time. So in summary, with the UASM, the appropriate range of sampling points to get the IP decay curve is $30-60$.

\section{Experiment}

Rock samples were obtained from a Chinese oilfield, and the sorting characteristics of clastic particles inside the rock samples are good or medium. Rock pores are mainly composed of intergranular dissolved pores, accounting for about $90 \%$, followed by primary intergranular pores and intragranular dissolved pores. The pore distributions are mainly double-peak or multimodal. For a double-peak sample A and a three-peak sample B, their porosity values are $30.2 \%$ and $24.5 \%$, and the 

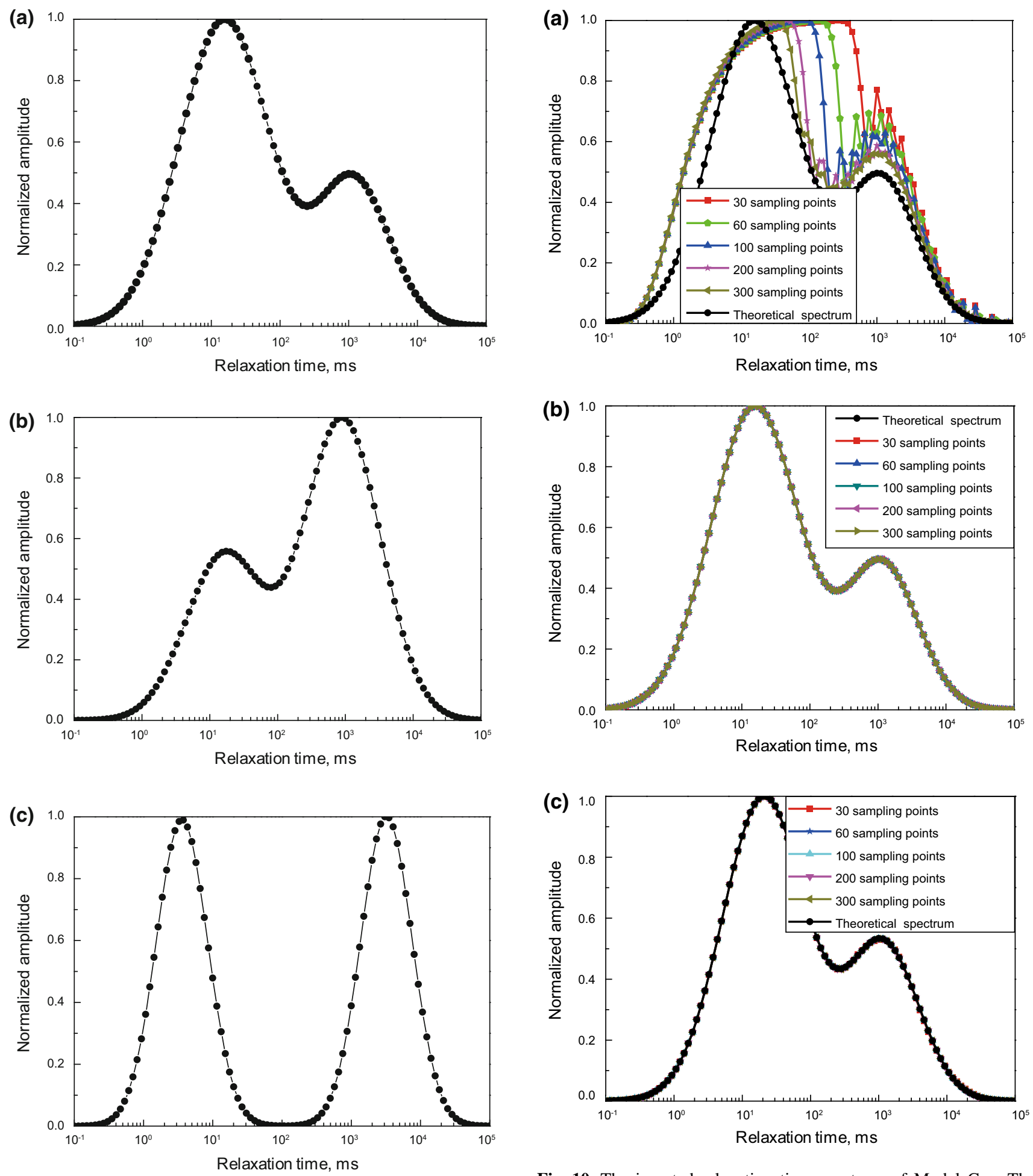

Fig. 9 Theoretical double-peak relaxation time spectrum models. a Model C, b Model D, c Model E

permeability values are $430 \times 10^{-3} \mu \mathrm{m}^{2}$ and $69.4 \times 10^{-3}$ $\mu \mathrm{m}^{2}$. The pore distributions of casting thin sections which were made from the rock samples were recorded

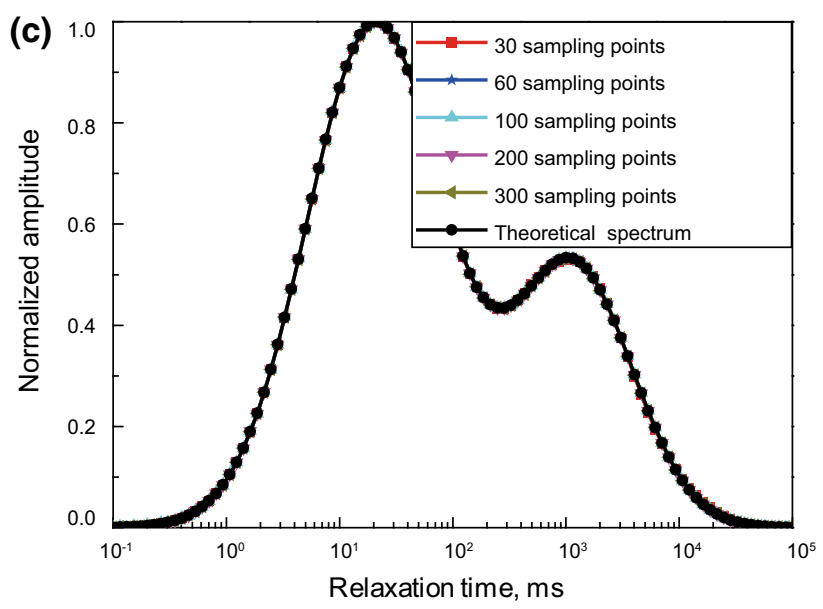

Fig. 10 The inverted relaxation time spectrum of Model C. a The relaxation time spectrum with the UTSM, b The relaxation time spectrum with the LTSM, c The relaxation time spectrum with the UASM

and are shown in Fig. 15, where the ordinate indicates the volume ratio of pores with a certain diameter to the total pores. 

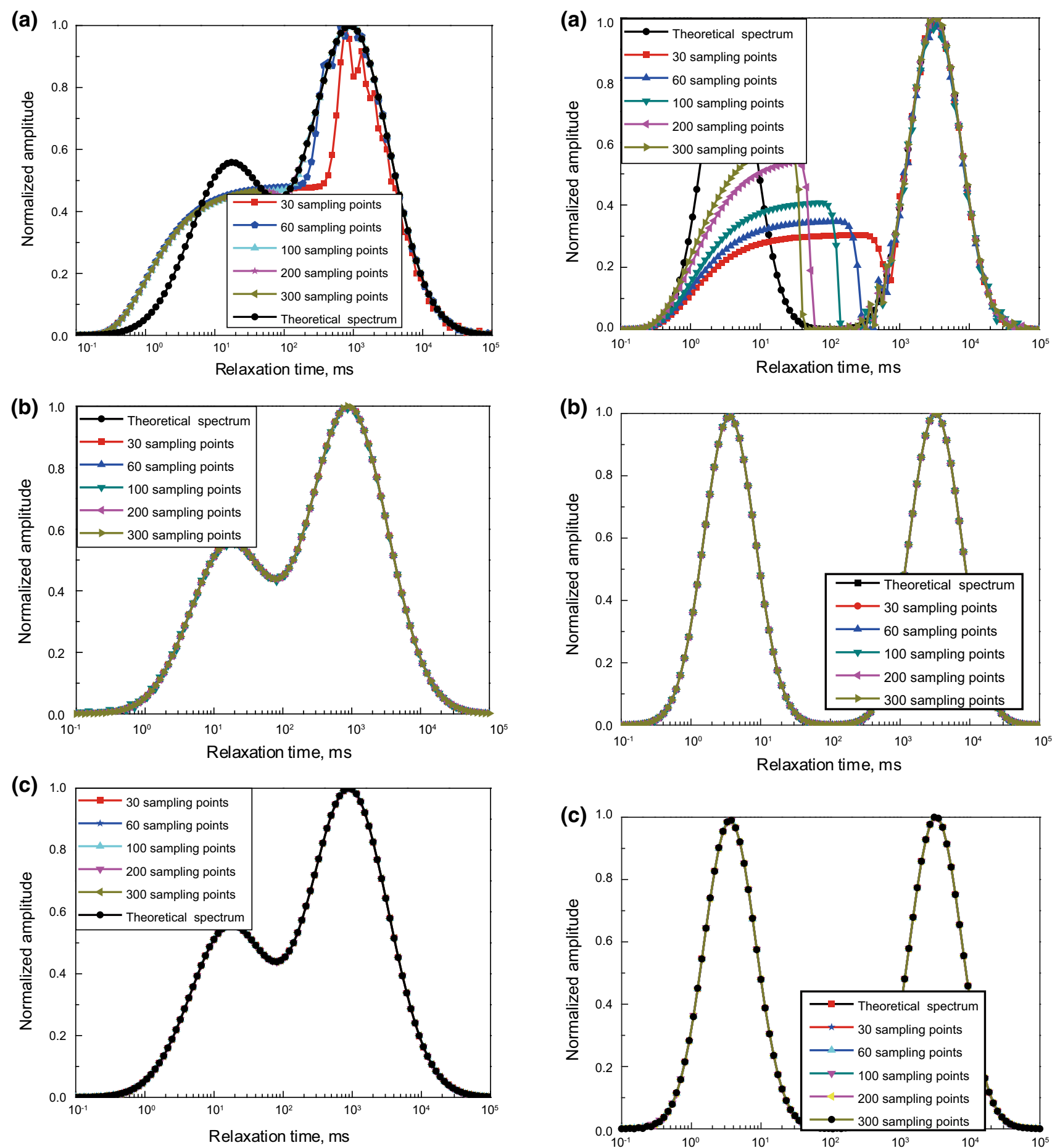

Fig. 11 The inverted relaxation time spectrum of Model D. a The relaxation time spectrum with the UTSM, b The relaxation time spectrum with the LTSM, c The relaxation time spectrum with the UASM

The pore diameter distribution can be transformed into RTS by Eq. (1), and based on it, the actual effect of the three sampling methods can be judged. We select 100

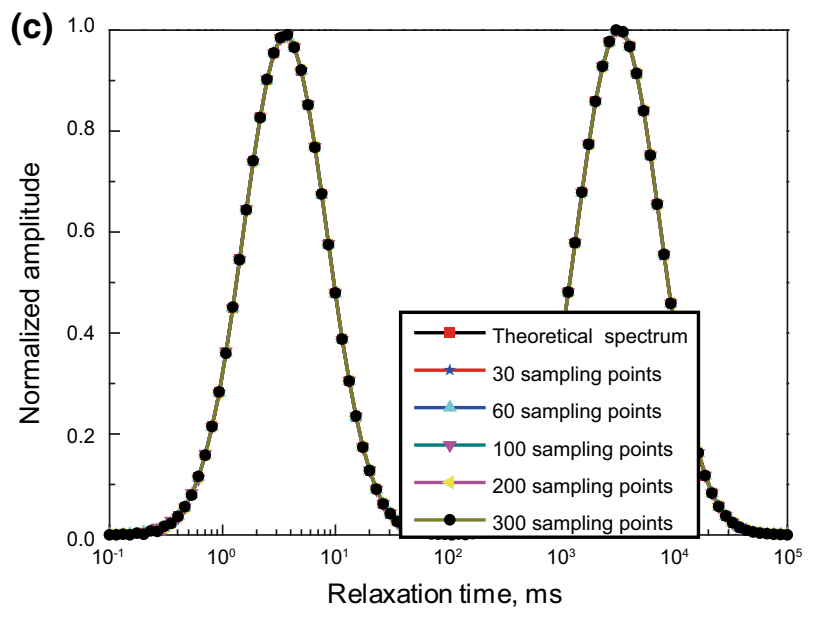

Fig. 12 The inverted relaxation time spectrum of Model E. a The relaxation time spectrum with the UTSM, b The relaxation time spectrum with the LTSM, c The relaxation time spectrum with the UASM

relaxation time constants following an even logarithmic distribution between $0.1 \mathrm{~ms}$ and $100,000 \mathrm{~ms}$, and then set the length of sampling time as $100,000 \mathrm{~ms}$ and the number 
Table 4 The RMSE of Model C's inversion with three methods

\begin{tabular}{llllll}
\hline Sampling points & 30 & 60 & 100 & 200 & 300 \\
\hline UTSM & 0.15 & 0.129 & 0.1 & 0.053 & 0.038 \\
LTSM & $1.13 \times 10^{-4}$ & $1.58 \times 10^{-5}$ & $4.26 \times 10^{-4}$ & $4.53 \times 10^{-4}$ & $7.55 \times 10^{-4}$ \\
UASM & $1.9 \times 10^{-3}$ & $6.2 \times 10^{-4}$ & $3.8 \times 10^{-4}$ & $4.4 \times 10^{-4}$ & $5.8 \times 10^{-4}$ \\
\hline
\end{tabular}

Table 5 The RMSE of model D's inversion with three methods
Table 6 The RMSE of model E's inversion with three methods

\begin{tabular}{llllll}
\hline Sampling points & 30 & 60 & 100 & 200 & 300 \\
\hline UTSM & 0.091 & 0.078 & 0.055 & 0.039 & 0.031 \\
LTSM & $1.13 \times 10^{-3}$ & $1.65 \times 10^{-4}$ & $1.34 \times 10^{-3}$ & & \\
$4.3 \times 10^{-4}$ & $2.1 \times 10^{-4}$ & & & \\
UASM & $8.5 \times 10^{-4}$ & $5.9 \times 10^{-4}$ & $7.5 \times 10^{-4}$ & & \\
$2.3 \times 10^{-4}$ & $1.8 \times 10^{-4}$ & & & & \\
\hline
\end{tabular}

\begin{tabular}{llllll}
\hline Sampling points & 30 & 60 & 100 & 200 & 300 \\
\hline UTSM & 0.271 & 0.262 & 0.248 & 0.215 & 0.195 \\
LTSM & $9.56 \times 10^{-5}$ & $5.61 \times 10^{-6}$ & $1.35 \times 10^{-3}$ & & $3.7 \times 10^{-4}$ \\
$1.25 \times 10^{-4}$ & & & & $5.1 \times 10^{-4}$ \\
UASM & $1.31 \times 10^{-3}$ & $5.11 \times 10^{-4}$ & $1.39 \times 10^{-3}$ & & \\
$7.8 \times 10^{-4}$ & & & & & \\
\hline
\end{tabular}

Table 7 Sampling time of model C, D, and E with UASM

\begin{tabular}{|c|c|c|c|c|c|}
\hline Sampling points & 30 & 60 & 100 & 200 & 300 \\
\hline odel C, ms & 12,259 & 19,121 & 24,250 & 28,462 & 32,087 \\
\hline Model D, ms & 8263 & 13,359 & 17,184 & 20,332 & 23,044 \\
\hline Model E, ms & 21,193 & 29,715 & 35,548 & 40,097 & 43,872 \\
\hline
\end{tabular}

of sampling points as 50 . The two rock samples were saturated with $3000 \mathrm{mg} / \mathrm{L} \mathrm{NaCl}$ solution. We cut the power off after sufficient power supply for the rock samples, and then the polarizability decay signal was obtained respectively with the three sampling methods. The inverted spectra are shown in Fig. 16. The sampling time of the three methods is shown in Table 8 .

Figure 16 shows clearly, with UTSM the inverted spectra have a large deviation with actual relaxation time spectra of the two samples. With LTSM and UASM, the inverted spectra are essentially coincident with actual spectra. Table 8 shows that sampling by using UASM takes only about half of the time lengths of the other two methods, so the vertical resolution can be improved effectively.

\section{Conclusions}

UASM can adapt well to measuring the strata with different kinds of pore size distribution. It has a good dynamic range for measuring both small pore size strata with fast decay IP voltage and large pore size strata with slow decay IP voltage with high RTS accuracy. A small number of sampling points from 30 to 60 are sufficient enough to get high RTS inversion accuracy thus the data traffic of the logging instrument is reduced effectively. UASM can 

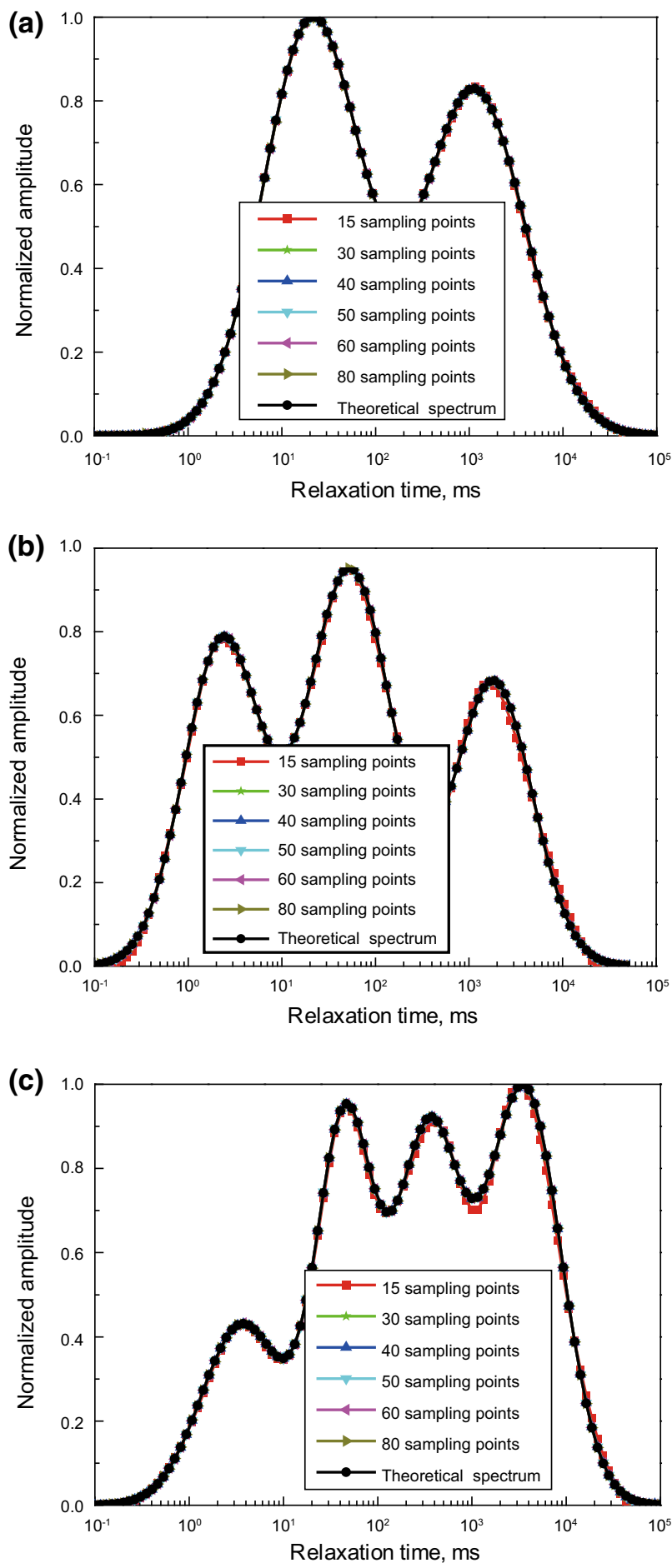

Fig. 13 The inverted spectra of the three models. a Model F, b Model G, c Model H

automatically adapt to the attenuation speed of the polarization curve, and the actual sampling time changes along with the rock pore size, which is helpful to improve the

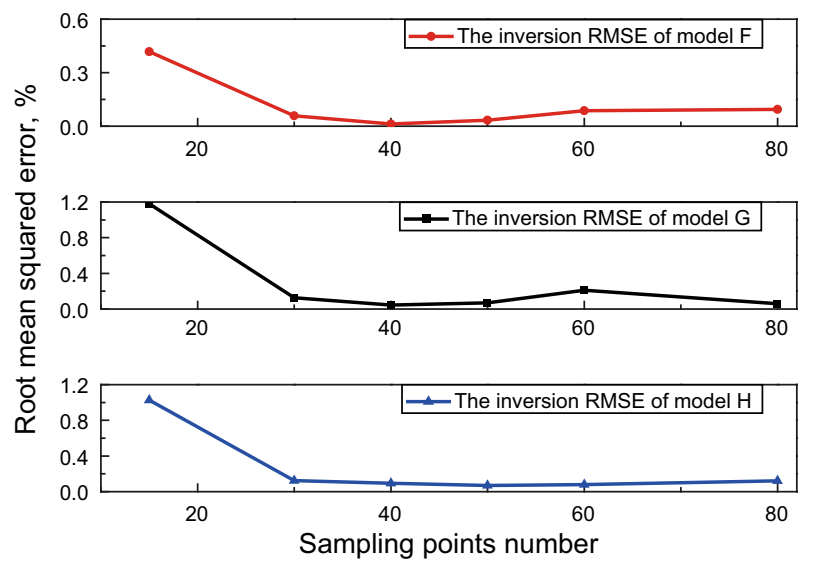

Fig. 14 The relationship between inversion RMSEs and sampling point numbers
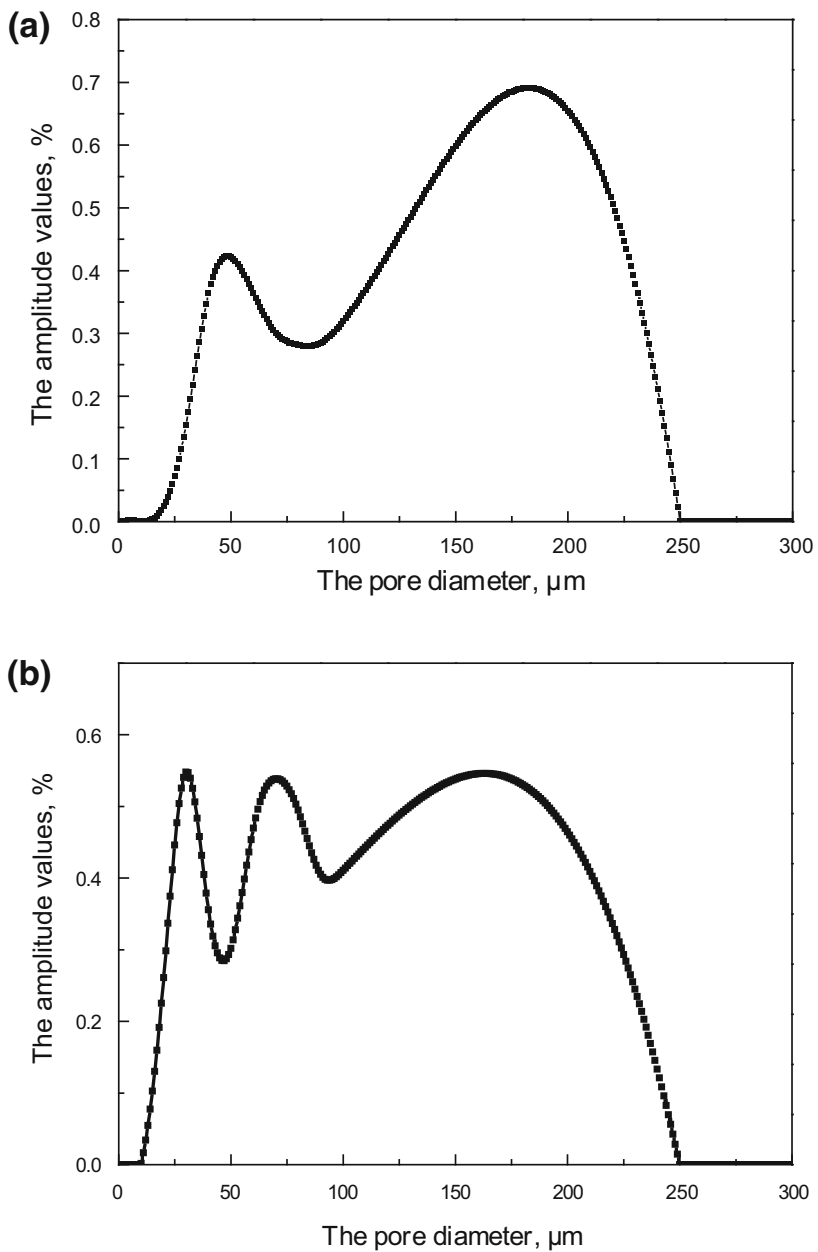

Fig. 15 The pore diameter distribution curve of the samples. a Sample A, b Sample B

vertical resolution of the strata measurement. The simulation and experimental results show that the UASM is superior to the UTSM and LTSM. 

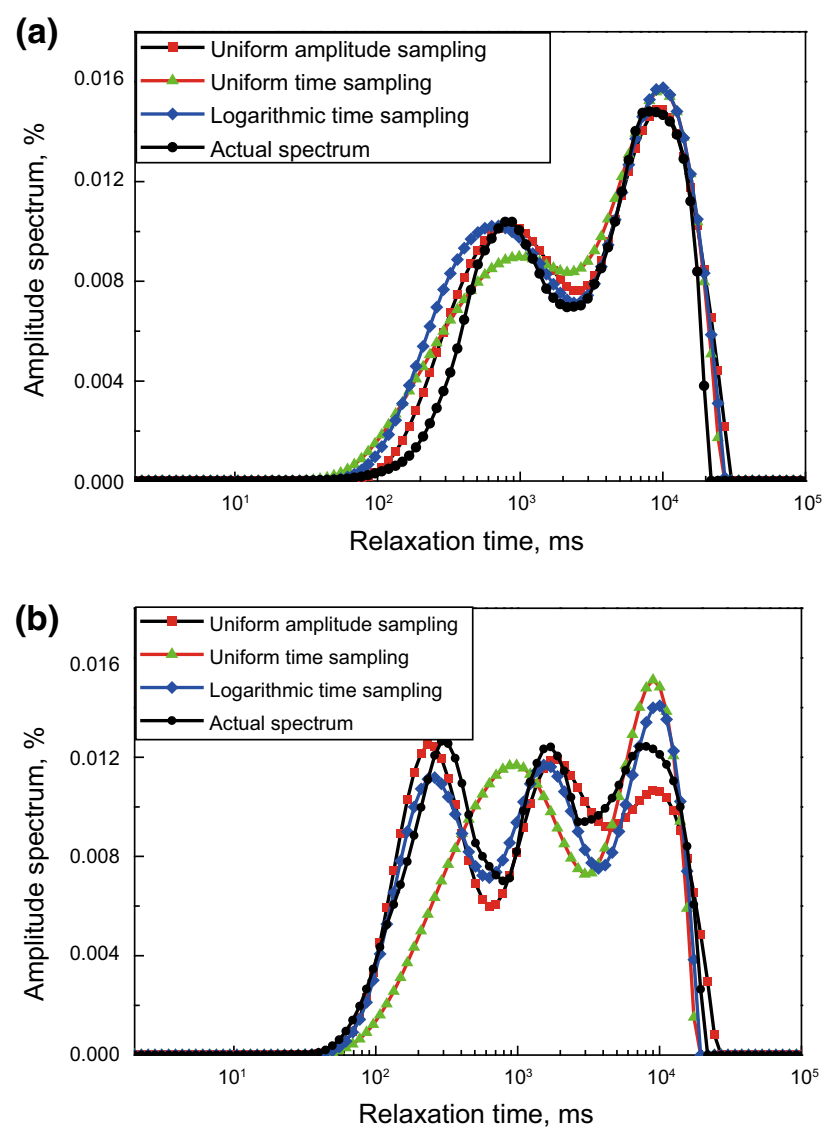

Fig. 16 The inverted relaxation time spectra of rock samples. a Rock sample A, b Rock sample B

Table 8 Sampling time of rock samples with the three sampling methods

\begin{tabular}{llll}
\hline Sampling time & UTSM & LTSM & UASM \\
\hline Sample A, ms & 100,000 & 100,000 & 58,802 \\
Sample B, ms & 100,000 & 100,000 & 56,720 \\
\hline
\end{tabular}

Acknowledgments This work was partially supported by a project from the National Natural Science Foundation of China (No. 61401168).

Open Access This article is distributed under the terms of the Creative Commons Attribution 4.0 International License (http://crea tivecommons.org/licenses/by/4.0/), which permits unrestricted use, distribution, and reproduction in any medium, provided you give appropriate credit to the original author(s) and the source, provide a link to the Creative Commons license, and indicate if changes were made.

\section{References}

Beckett CTS, Augarde CE. Prediction of soil water retention properties using pore-size distribution and porosity. Can Geotech J. 2013;50(4):435-50.
Binley A, Slater LD, Fukes M, et al. Relationship between spectral induced polarization and hydraulic properties of saturated and unsaturated sandstone. Water Resour Res. 2005;41(12):W12417.

Buecker M, Hoerdt A. Long and short narrow pore models for membrane polarization. Geophysics. 2013;78(6):299-314.

Chen SS, Wang HZ, Zhang XL. Study on multi-exponential inversion method for NMR relaxation signals with Tikhonov regularization. Engineering. 2013;5(1):32-7.

Florsch N, Revil A, Camerlynck C. Inversion of generalized relaxation time distributions with optimized damping parameter. J Appl Geophys. 2014;109:119-32.

Guan JT, Wang Q, Fan YH, et al. Study on the mechanisms of electrochemical logging response in shaly sandstone based on capillary model. Chin J Geophys. 2010;53(1):214-23 (in Chinese).

Gurin G, Tarasov A, Ilyin Y, et al. Time domain spectral induced polarization of disseminated electronic conductors: Laboratory data analysis through the Debye decomposition approach. J Appl Geophys. 2013;98:44-53.

Jang H, Park S, Kim HJ. A simple inversion of induced-polarization data collected in the Haenam area of Korea. J Geophys Eng. 2014;11(1):015011.

Li CL, Zhou CC, Li X, et al. A novel model for assessing the pore structure of tight sands and its application. Appl Geophys. 2010;7(3):283-91.

Li Y, Lin PR, Xiao Y, et al. Induced polarization effect on frequency domain electromagnetic sounding with electric dipole source. Chin J Geophys. 2011;54(7):1935-44 (in Chinese).

Liu HQ, Yan J, Deng YM. Study of the properties of non-gas dielectric capacitors in porous media. Pet Sci. 2015;12(1):104-13.

Liu XN, Kong L, Zhang P, et al. Permeability estimation using relaxation time spectra derived from differential evolution inversion. J Geophys Eng. 2014a;11(1):1-8.

Liu XN, Kong L, Zhou KB, et al. A time domain induced polarization relaxation time spectrum inversion method based on a damping factor and residual correction. Pet Sci. 2014b;11(4):519-25.

Moody JB, Xia Y. Analysis of multi-exponential relaxation data with very short components using linear regularization. J Magn Reson. 2004;167(1):36-41.

Nie XW, Zhou AC, Yao GD, et al. Prospecting for oil and gas with induced polarization method-a discussion on the effect and anomaly model. Chin J Geophys. 1987;30(4):412-22.

Revil A, Florsch N, Camerlynck C. Spectral induced polarization porosimetry. Geophys J Int. 2014;198(2):1016-33.

Rezaee MR, Jafari A, Kazemzadeh E. Relationships between permeability porosity and pore throat size in carbonate rocks using regression analysis and neural networks. J Geophys Eng. 2006;3(4):370-6.

Shou JF, Zhu GH. Study on quantitative prediction of porosity preservation in sandstone reservoirs. Chin J Geol (Sci Geol Sin). 1998;32(2):244-50 (in Chinese).

Tan FQ, Li HQ, Xu CF, et al. Quantitative evaluation methods for waterflooded layers of conglomerate reservoir based on well logging data. Pet Sci. 2010;7(4):485-93.

Titov K, Komarov V, Tarasova V, et al. Theoretical and experimental study of time domain-induced polarization in water-saturated sands. J Appl Geophys. 2002;50(4):417-33.

Titov K, Tarasov A, Ilyin Y, et al. Relationships between induced polarization relaxation time and hydraulic properties of sandstone. Geophys J Int. 2010;180(3):1095-106.

Tong MS, Li L, Wang W, et al. Estimation of pore size distribution and permeability of shaly sands from induced polarization time spectra. Chin J Geophys. 2005;48(3):785-91 (in Chinese).

Tong MS, Tao HG. Experimental study of induced polarization relaxation time spectra of shaly sands. J Pet Sci Eng. 2007;59(3-4):239-49. 
Tong MS, Wang WN, Jiang YZ, et al. Estimation of permeability of shaly sand reservoir from induced polarization relaxation time spectra. J Pet Sci Eng. 2004;45(1-2):31-40.

Wang CJ. Induced polarization potential logging technique. Spec Oil Gas Reservoirs. 2004; 11(4): 57-9, 66 (in Chinese).

Wang WN, Yu QF, Jian YZ, et al. Induced polarization potential attenuation spectrum well logging continuous recording method. OGP. 2010;45(Supplement 1):214-6 (in Chinese).

Wang WN, Yu QF, Tong MS, et al. Electrode configuration design of induced polarization potential decay spectrum logging. Progr Geophys. 2011;26(1):371-5 (in Chinese).

Wu RQ, Chen W, Chen TQ, et al. A time-driven transmission method for well logging networks. Pet Sci. 2009;6(3):239-45.

Xiao LZ, Zhang HR, Liao GZ, et al. Inversion of NMR relaxation in porous media based on Backus-Gilbert theory. Chin J Geophys. 2012;55(11):3821-8 (in Chinese).
Yang RF, Wang Y, Cao J. Cretaceous source rocks and associated oil and gas resources in the world and China: a review. Pet Sci. 2014;11(3):331-45.

Zhang W, Shi YB, Tang JY. Application of an improved ADSL technique in the high-speed wireline logging telemetry system. ICEMI. 2007;2:108-12.

Zhu XM, Zhu SF, Xian BZ, et al. Reservoir differences and formation mechanisms in the Ke-Bai overthrust belt, northwestern margin of the Junggar Basin, China. Pet Sci. 2014;7(1):40-8.

Zimmermann E, Kemna A, Berwix J, et al. A high-accuracy impedance spectrometer for measuring sediments with low polarizability. Meas Sci Technol. 2008;19(10):105603. 